\title{
Evidencias del origen de una posible nueva entidad de Dioscorea (Dioscoreaceae) de las Sierras de Calilegua, Jujuy (Argentina). Un enfoque morfo-anatómico
}

\author{
Evidence of the origin of a possible new entity of Dioscorea \\ (Dioscoreaceae) from Sierras de Calilegua, Jujuy (Argentina). \\ A morpho-anatomical approach
}

\footnotetext{
Asesor, Patricia N. ${ }^{\text {; }}$ Patricia L. Albornoz ${ }^{2,3}{ }^{*}$; Eva Bulacio ${ }^{1}$

1 Laboratorio de Taxonomía Fanerogámica, Fundación M. Lillo. Miguel Lillo 251, (T4000JFE) San Miguel de Tucumán, Tucumán, Argentina.

2 Instituto de Morfología Vegetal, Fundación M. Lillo. Miguel Lillo 251, (T4000JFE) San Miguel de Tucumán, Tucumán, Argentina.

3 Cátedra de Anatomía Vegetal, Facultad de Ciencias Naturales e IML, Universidad Nacional de Tucumán. Miguel Lillo 205, (T4000JFE) San Miguel de Tucumán, Tucumán, Argentina.

* Autor corresponsal: plalbornoz@lillo.org.ar
}

\section{RESUMEN}

En las Sierras de Calilegua reconocimos siete especies de Dioscorea como parte de su flora y una octava entidad que denominamos Dioscorea sp., la cual evidenció algunos caracteres morfológicos similares a $D$. glomerulata y $D$. haumanii, posibles progenitores de ésta, dado que comparten el área de distribución en zonas propicias para el fenómeno de hibridación. El objetivo del presente trabajo fue analizar la morfología floral y la morfoanatomía foliar de D. glomerulata, D. haumanii y Dioscorea sp. Se trabajó con material fresco y herborizado al que se aplicaron técnicas convencionales. Los resultados evidenciaron que los caracteres morfoanatómicos de Dioscorea sp. no son intermedios a los de sus probables progenitores. El análisis de componentes principales permitió agrupar a los individuos de cada entidad por separado. Por

\footnotetext{
Ref. bibliográfica: Asesor, P. N.; Albornoz, P. L.; Bulacio, E. 2019. "Evidencias del origen de una posible nueva entidad de Dioscorea (Dioscoreaceae) de las Sierras de Calilegua, Jujuy (Argentina). Un enfoque morfo-anatómico". Lilloa 56 (2): 1-17. Fundación Miguel Lillo, Tucumán, Argentina. D.0.I.: doi.org/10.30550/ j.lil/2019.56.2/1

Recibido: 12/09/18 - Aceptado: 04/07/19

$>$ URL de la revista: http://lilloa.lillo.org.ar

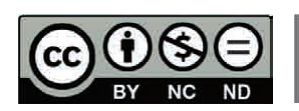

> Algunos derechos reservados. Esta obra está bajo una Licencia Creative Commons Atribución - No Comercial - Sin Obra Derivada 4.0 Internacional.
} 
esta razón, podemos concluir que este estudio posiciona a Dioscorea sp. en un lugar estable e independiente.

Palabras clave - Anatomía, Dioscorea, flor, hoja, morfología.

\section{ABSTRACT}

In the Sierras de Calilegua we recognized seven species of Dioscorea as part of their flora and an eighth entity that we named Dioscorea sp., which showed some morphological features similar to $D$. glomerulata and $D$. haumanii, possible progenitors of this, since they share the area of distribution in zones conducive to the phenomenon of hybridization. The aim of the present work was to analyze the floral morphology and leaf morphoanatomy of D. glomerulata, D. haumanii and Dioscorea sp. We worked with fresh and herborized material to which conventional techniques were applied. The results showed that the morphoanatomical characteristics of Dioscorea sp. are not intermediate to those of their probable parents. Principal components analysis allowed to group individuals of each entity separately. For this reason, we can conclude that this study places Dioscorea sp. in a stable and independent position.

Keywords - Anatomy, Dioscorea, flower, leaf, morphology.

\section{INTRODUCCIÓN}

En el marco del estudio que llevamos adelante desde 2009 sobre Dioscorea L. (Dioscoreaceae $\mathrm{R}$. Br.) que habitan en las montañas del noroeste argentino, realizamos numerosas campañas de recolección a las Sierras de Calilegua, en la provincia de Jujuy. En ellas reconocimos siete especies, D. glomerulata Hauman, D. hassleriana Chodat, D. dodecaneura Vell., D. glandulosa Klotzch ex Kunth, D. haumanii Xifreda, $D$. cieneguensis $\mathrm{R}$. Knuth, $D$. spectabilis $\mathrm{R}$. Knuth y una octava, a la que denominamos Dioscorea sp. Esta última presentó como carácter morfológico singular, la presencia de un estambre único, condición que presenta también Dioscorea monandra Hauman. Esta es una especie controversial descripta a principios del siglo pasado por Hauman (1916), quien le asignó el epíteto específico en referencia a la presencia de un único estambre, el cual fue interpretado como una reducción del patrón floral del género. Cabe destacar que posteriores interpretaciones (Xifreda, 1996; Govaerts, Wilkin, Saunders, 2007) consideraron a este aspecto un estadio intermedio entre especies próximas, razón por lo cual postularon un origen híbrido para la especie. Sin embargo, no se encontró ningún estudio que respalde específicamente tal postulado.

Dioscorea sp. difiere sustancialmente de $D$. monandra por la estructura de la columna estaminal. A pesar de estas diferencias, de no compartir el área de distribución y de mantener los caracteres morfológicos estables a lo largo de los años, el estambre único de Dioscorea sp. podría llevar a diferentes interpretaciones, entre ellas un origen híbrido, similar a lo ocurrido con D. monandra. 
La hibridación se manifiesta principalmente por el reconocimiento de caracteres morfológicos intermedios (Rushton, 1993) para lo cual es necesario conocer completamente las características morfológicas y anatómicas presentes en el fenotipo de cada especie parental (Zobel y Talbert, 1994). En este contexto, consideramos que las probables especies progenitoras de Dioscorea sp. serían D. glomerulata y D. haumanii. Las tres entidades comparten caracteres morfológicos, además del área de distribución, específicamente zonas de ecotono y sometidas a disturbios, características de áreas propicias para el intercambio genético entre diferentes especies (Barton y Hewitt, 1985). En cuanto a los antecedentes anatómicos, para la familia se cuenta con revisiones generales que incluyen estudios de la hoja, tallo y raíz de especies del Viejo Mundo (Burkill, 1960; Ayensu, 1972; Huber, 1998). Abdulrahaman, Egbedo, Oladele (2009); Daniel y Atumeyi (2011); Marín, Vargas, Oropeza (2012), evaluaron la anatomía foliar de especies de valor alimenticio de Asia y Oceanía. En Sudamérica, Xifreda $(1990,2000)$ sugirió utilizar, como elementos de valor diagnóstico, la presencia de la vaina cristalífera en el tallo y glándulas en la hoja, para diferenciar especies, mientras que Barroso et al. (1974) examinaron la anatomía foliar y floral de las dioscoreas de la flora de Brasil.

El objetivo de este trabajo fue analizar los aspectos morfológicos de la flor y morfoanatómicos foliares, con el fin de interpretar el grado de relación entre $D$. glomerulata, D. haumanii y Dioscorea sp. de las Sierras de Calilegua.

\section{MATERIALES Y MÉTODOS}

El material estudiado proviene de las Sierras de Calilegua, un cordón de más de $3.000 \mathrm{~m}$ de altura perteneciente al sistema de Sierras Subandinas, ubicado en el centro-norte de la provincia de Jujuy, Argentina. Las características climáticas de estas sierras son las propias del noroeste argentino, con una marcada estacionalidad especialmente en las precipitaciones, las que ocurren mayoritariamente en verano y, según la altitud, alcanzan valores de entre 1.000 a $2.000 \mathrm{~mm}$ al año (Ayarde, 2013). La vegetación, correspondiente a la provincia fitogeográfica de las Yungas (Cabrera, 1976), se dispone en fajas altitudinales que van de bosques deciduos pedemontanos hasta bosques y pastizales montanos (Bulacio y Ayarde, 2009; Ayarde, 2013).

Los ejemplares estudiados corresponden a D. glomerulata, D. haumanii y Dioscorea sp. De las tres se realizaron observaciones «in situ» y de las dos primeras especies además se revisaron ejemplares depositados en herbarios de referencia de Argentina (BA, BAF, CORD, LIL y SI) y herbarios virtuales (BGBM, G, K, M, MO, US). Parte de los ejemplares herborizados fueron depositados en el Herbario de la Fundación Miguel Lillo. Se utilizó material fresco y fijado en FAA (1:1:8, v/v/v formol, ácido acético glacial, etanol $80 \%$ ).

Las especies estudiadas se diferencian básicamente por la morfología de su inflorescencia y por la estructura de la flor masculina.

Dioscorea glomerulata es una especie dioica. La inflorescencia es solitaria, geminada o acompañada de una rama foliar, de 5 a $20 \mathrm{~cm}$ de largo; las flores están reunidas en glomérulos plurifloros (de 5 a 8) en la base y paucifloros ( 2 a 3) en el ápice; tiene 
6 estambres libres, insertos en la base del perianto hasta la mitad de su longitud, con filamentos filiformes, un cuarto más largos que la antera. Habita ambientes de Yungas en las provincias de Catamarca, Tucumán, Salta y Jujuy y por lo común se la encuentra en áreas abiertas o con alguna modificación antrópica, desde la zona de contacto con la vegetación chaqueña (Xifreda, 2009) hasta bosques y arbustales montanos por encima de los $2.000 \mathrm{~m}$ (Asesor y Bulacio, 2017). En las Sierras de Calilegua habita entre los 1.200 y $2.800 \mathrm{~m}$ snm.

Dioscorea haumanii es una especie monoica. La inflorescencia masculina está compuesta por racimos multifloros de 12 a $20 \mathrm{~cm}$ de long., solitarios, de a 4 o más; las flores están aisladas, desde casi opuestas a distanciadas 7 a $15 \mathrm{~mm}$, los tépalos son purpúreos, oval-lanceolados, subiguales, trinervados, el cuerpo estaminal está formado por un pie corto, costado, de donde nacen 3 proyecciones corniculiformes semi-carnosas, de ápice apenas retorcido, base ancha y bordes externos pilosos; tiene 3 anteras, sésiles, adosadas al eje central alternando con las proyecciones laminares. Posee rudimento estilar. Se distribuye en las provincias de Salta y Jujuy, en el distrito Chaco serrano subhúmedo (Xifreda, 2009) y en zona de transición con Yungas (Asesor y Bulacio, 2017). En las Sierras de Calilegua se la encuentra entre los 1.400 y $1.600 \mathrm{~m}$ snm.

Dioscorea sp. es monoica. Tiene racimos axilares pubérulos, de $5-10 \mathrm{~cm}$; la flor posee perianto estrellado; tépalo con centro purpúreo y bordes blanco-verdosos, angostamente ovado, trinervado, con abundantes pelos eglandulares en la base de la cara interna; la columna estaminal es corta, ca. $1 \mathrm{~mm}$, ensanchada en su parte apical en un cuerpo navicular terminado en tres proyecciones dendriformes, la parte central púrpura y las laterales blanco-verdosas, cubiertas de largos pelos eglandulares; la antera es única, con línea de dehiscencia evidente; el rudimento estilar es nulo. Hasta el presente sólo se la ha encontrado en las Sierras de Calilegua donde habita desde los 1.800 hasta los $2.900 \mathrm{~m}$ snm, en bosque y pastizal montano (Asesor y Bulacio, 2017).

Para la evaluación de los caracteres morfológicos y anatómicos se recolectaron cinco individuos al azar de cada entidad, tomándose al azar 10 flores masculinas de cada individuo para la caracterización floral y 10 hojas maduras y sanas para la caracterización foliar.

El análisis de la epidermis y venación foliar se realizó mediante diafanizados según la técnica de Dizeo de Strittmater (1973). Para la caracterización estructural se hicieron cortes transversales de la porción media de la lámina, con la técnica de «mano libre» (D’Ambrogio de Argüeso, 1986). Los colorantes usados fueron cristal violeta y una doble tinción de azul astra-safranina y, como medio de montaje, se utilizó agua-glicerina (1:1). En la descripción de la venación foliar se siguió la terminología de Hickey $(1974,1979)$ y Ellis et al. (2009). Se empleó la terminología propuesta por Dilcher (1974) para la caracterización de los estomas, aun tratándose de Monocotiledóneas, ya que los estomas presentan formas y características semejantes a los de Dicotiledóneas. De cada muestra foliar se analizaron cinco campos ópticos en los que se determinó la densidad (estomas $\left./ \mathrm{mm}^{2}\right)$, tamaño de estomas $(\mu \mathrm{m})$ y tamaño de tricomas eglandulares y glandulares $(\mu \mathrm{m})$.

Las observaciones se realizaron con microscopio óptico (Zeiss, Axiostar Plus, 
Göttingen, Germany) y lupa (Olympus, SZX7, Japan). Las fotografías fueron tomadas con cámara digital (Canon A620, Power Shot 7,1 MP y Olympus Sp-350 d $8 \mathrm{MP}$ ). La identificación de cristales se determinó utilizando el microscopio de luz polarizada (Zeiss, AXIO, Lab A1, Göttingen, Germany).

\section{Análisis de Componentes Principales (ACP)}

Para el análisis estadístico se emplearon caracteres morfológicos florales y foliares y anatómicos foliares. La matriz básica se elaboró con 25 muestras correspondientes a cinco hojas de cada una de las tres entidades (total de 75 muestras) y seis variables morfológicas y anatómicas: tamaño de la hoja (TH), diámetro de la flor masculina (DFM), número de estambres por flor (ExF), tamaño de estomas (TE), densidad estomática (DE) y tamaño del tricoma glandular (TTG). Se empleó un Análisis de Componentes Principales (ACP) con la intención de explorar la relación entre los ejemplares de las tres entidades y las variables, y en consecuencia de qué manera se ordenan los mismos. Esta metodología multivariada posibilita reducir la variabilidad de los datos con la menor pérdida de información, al transformar las variables originales en componentes principales, o sea un conjunto de variables no correlacionadas. El diagrama sintetiza la estructura de los datos, donde las entidades están representadas por puntos y las variables por vectores dentro de un sistema de coordenadas cartesianas conformadas por los dos primeros ejes o componentes. Las proximidades entre los puntos sugieren similitud de comportamiento de las entidades respecto a las variables. Por su parte las mismas se unen en el centro del gráfico y dan una idea de cuan relacionadas están entre sí o entre los componentes. Cuanto más agudo sea el ángulo entre variables y el componente, la correlación es mayor y por lo tanto mayor será su contribución a la formación de ese componente, si el ángulo es de $90^{\circ}$ la correlación es nula y si es obtuso la correlación es inversa.

Para realizar el análisis se utilizó el programa estadístico InfoStat Versión 2012 (Di Rienzo et al., 2012).

\section{Material examinado}

Dioscorea glomerulata Hauman. ARGENTINA. Prov. Jujuy, Dpto. Valle Grande, Quebrada de Cortaderas, 9-XII-2012, Bulacio y Ayarde 2504 (LIL); ibid., senda al Alto Calilegua, Abra de Duraznillo, 24-II-2015, Asesor, Buedo, Ayarde y Bulacio 120 (LIL); ibid., Pino de descanso, 24-II-2015, Asesor, Buedo, Ayarde y Bulacio 119 (LIL); ibid., entrada Quebrada de Cortaderas, 26-II-2015, Asesor, Buedo, Ayarde y Bulacio 128 (LIL); ibid., Quebrada de Cortaderas, 26-II-2015, Asesor, Buedo, Ayarde y Bulacio 129 (LIL).

Dioscorea haumanii Xifreda. ARGENTINA. Prov. Jujuy, Dpto. Ledesma, Rio San Lorenzo, próximo a Ledesma, 25-II-1940, Burkart y Troncoso 11257 (SI); ibid., Dpto. Valle Grande, a $1 \mathrm{Km}$ de Valle Grande, 26-III-2016, Andrada A. R., Ayarde H. 
y Bulacio E. 10 (LIL); ibid., Valle Grande, 26-III-2016, Andrada A. R., Ayarde H. y Bulacio E. 11 (LIL); ibid., entre San Francisco y Valle Grande, 12-I-2017, Bulacio E. 2726 (LIL); ibid., $1 \mathrm{Km}$ pasando Valle Grande, camino a Valle Colorado, 12-I-2017, Bulacio E. 2728 (LIL)

Dioscorea sp. ARGENTINA. Prov. Jujuy, Dpto. Valle Grande, Aguas Blancas, senda al Alto Calilegua, pasando Qda. Honda, 25-II-2009, Ayarde y Bulacio 1600 (LIL); ibid., Tolditos, senda al Alto Calilegua, 10-XII-2012, Ayarde y Bulacio 2585 (LIL); ibid., Abra de Duraznillo, 24-II-2015, Asesor, Buedo, Ayarde y Bulacio 115 (LIL); ibid., Pino Hachado, 24-II-2015, Asesor, Buedo, Ayarde y Bulacio 112 (LIL); ibid., Quebrada de Cortaderas, 26-II-2015, Asesor, Buedo, Ayarde y Bulacio 127 (LIL).

\section{RESULTADOS}

\section{Arquitectura foliar}

(Fig. 1, Tabla 1)

En las tres entidades estudiadas la hoja es ovada, membranácea y de margen entero; simétrica o asimétrica (Fig. 1D-F, Tabla 1). La venación es campilódroma con siete a nueve venas primarias, débiles a moderadas, originadas en un punto y formando arcos que convergen en el ápice (Fig. 1D-F). Las venas secundarias son delgadas a capilares o moderadas a capilares (Fig. 1G-I, Tabla 1), con ángulo de divergencia agudo. En D. glomerulata y D. haumanii se observan venas intersecundarias (Fig. 1G, $\mathrm{H})$. Todas presentan venas terciarias de modelo reticulado ortogonal que delimitan areolas de desarrollo imperfecto (Fig. 1G-I) que pueden o no contener vénulas, en este caso simples o ramificadas (Fig. 1K, L; Tabla 1); la venación última es marginal fimbriada (Fig. 1J).

\section{Anatomía foliar}

(Fig. 2, Tabla 2)

En vista paradermal las tres entidades presentan epidermis adaxial con células isodiamétricas de paredes rectas a curvas (Fig. 2A-C), en D. glomerulata, además, se observan células rectangulares a cuadrangulares (Fig. 2A, Tabla 2). La epidermis abaxial presenta células isodiamétricas (Fig. 2D, E), excepto en Dioscorea sp. donde las células son rectangulares (Fig. 2F, Tabla 2). Los estomas se ubican en la superficie abaxial, elevados o al mismo nivel de las células epidérmicas (Tabla 2), son principalmente anomocíticos (Fig. 2G), raro polocíticos (Fig. 2H), cuyos tamaños varían entre 1,29 $+/-0,07$ a $1,38+/-0,10 \mu \mathrm{m}$ (Tabla 2 ), además hay presencia de estomas gemelos (Fig. 2I). Los tricomas son de tipo eglandular unicelular y glandular pluricelular. Los primeros son cortos (Fig. 2J) y largos (Fig. 2K) que pueden o no ramificarse (Fig. $2 \mathrm{~K}$, L, Tabla 2). Los tricomas glandulares presentan un pie unicelular y una cabeza 

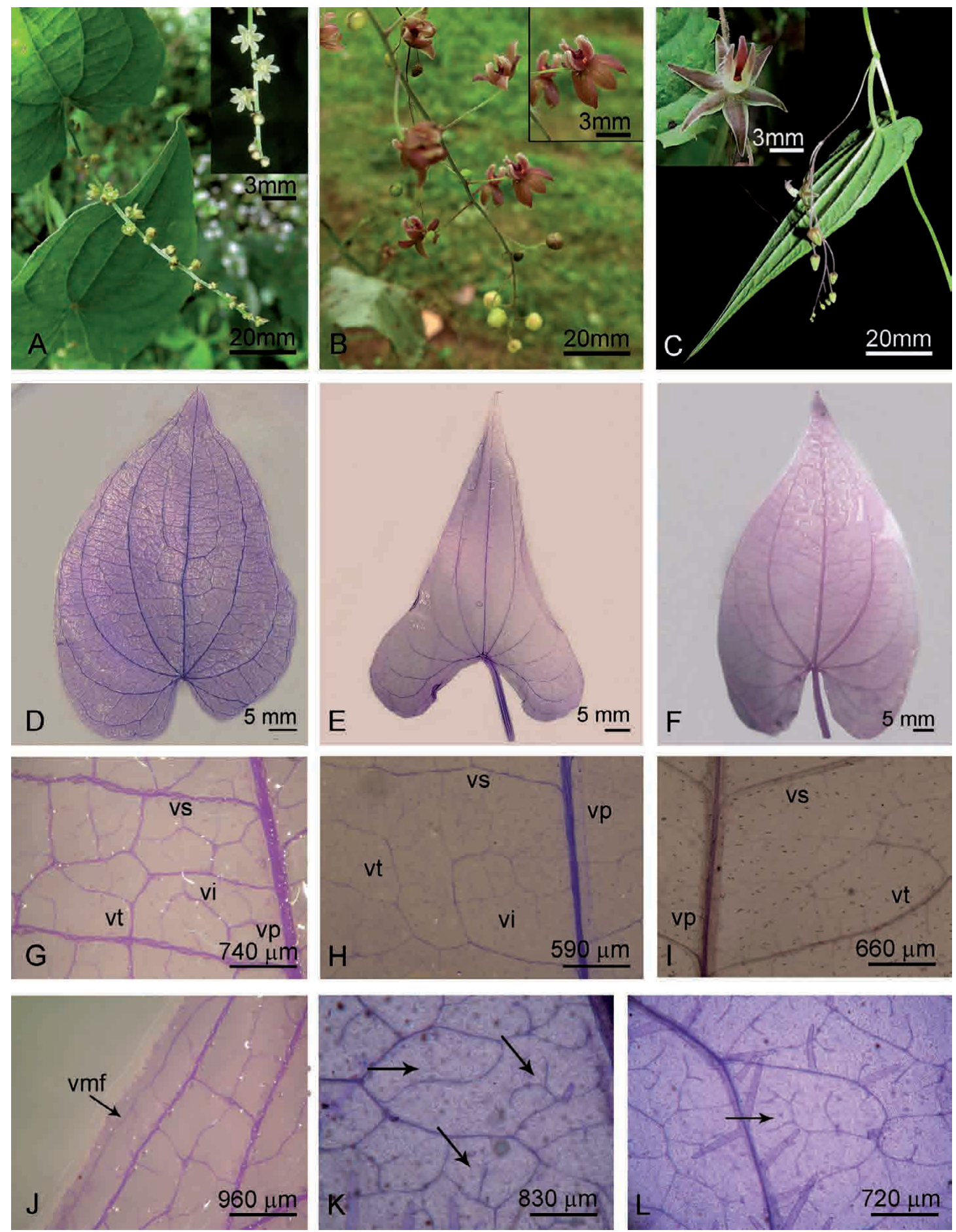

Fig. 1. Aspecto de la inflorescencia masculina y arquitectura foliar. A, D, G) Dioscorea glomerulata. B, E, H) Dioscorea haumanii. C, F, I) Dioscorea sp. A-C) Aspecto de la inflorescencia masculina. D-F) Aspecto de la lámina mostrando la venación. G-I) Detalle de la venación. J) Detalle de la vena marginal fimbriada. K, L) Detalle de areolas y vénulas. Referencias: vi, vena intersecundaria; vp, vena primaria; vs, vena secundaria; vt, vena terciaria. Andrada A. R., Ayarde H. y Bulacio E. 10 (LIL), Asesor, Buedo, Ayarde y Bulacio 127 (LIL). 
Tabla 1. Caracteres diferenciales de la morfología floral masculina y de la morfología y arquitectura foliar de D. glomerulata, D. haumanii y Dioscorea sp.

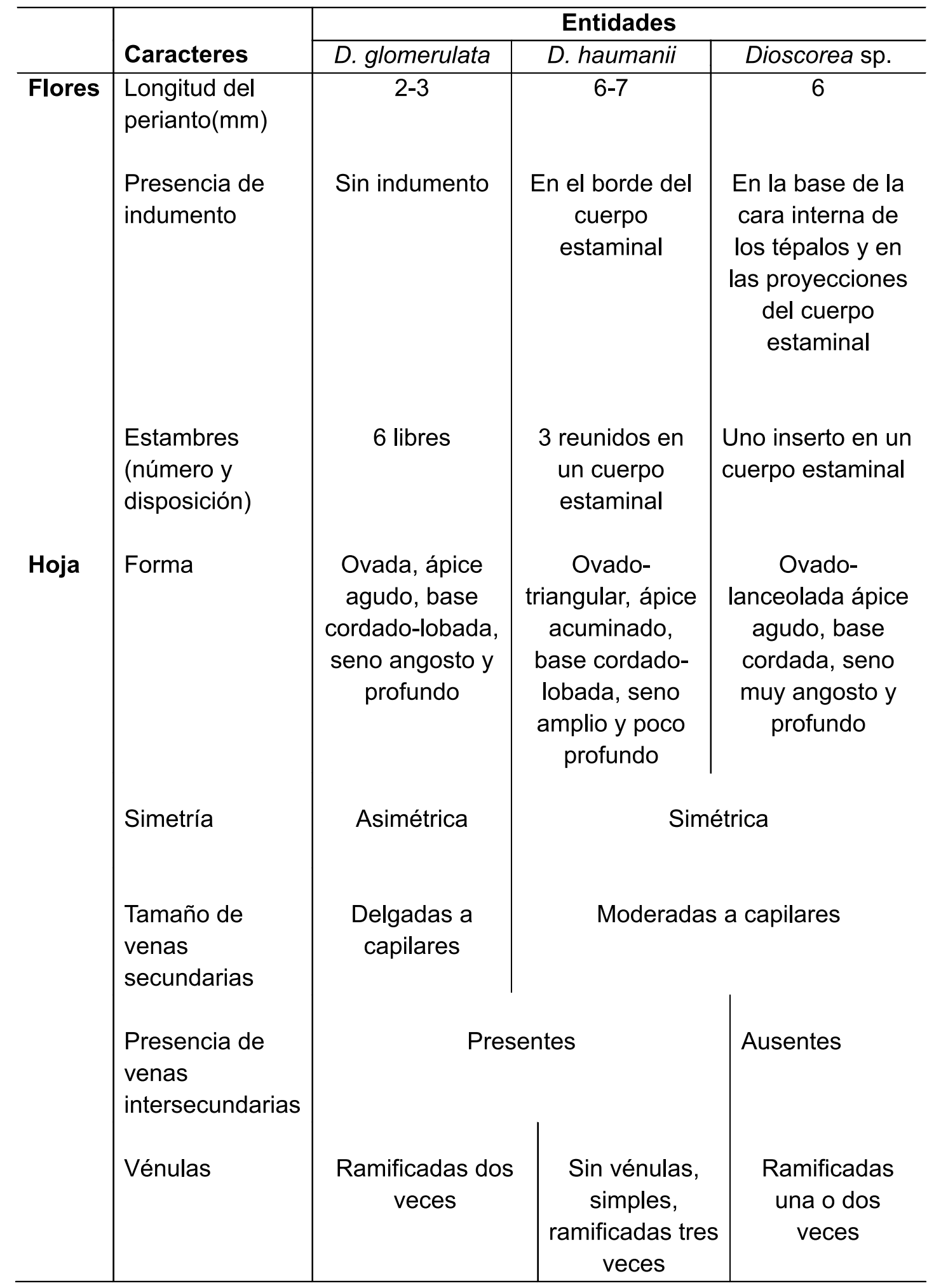



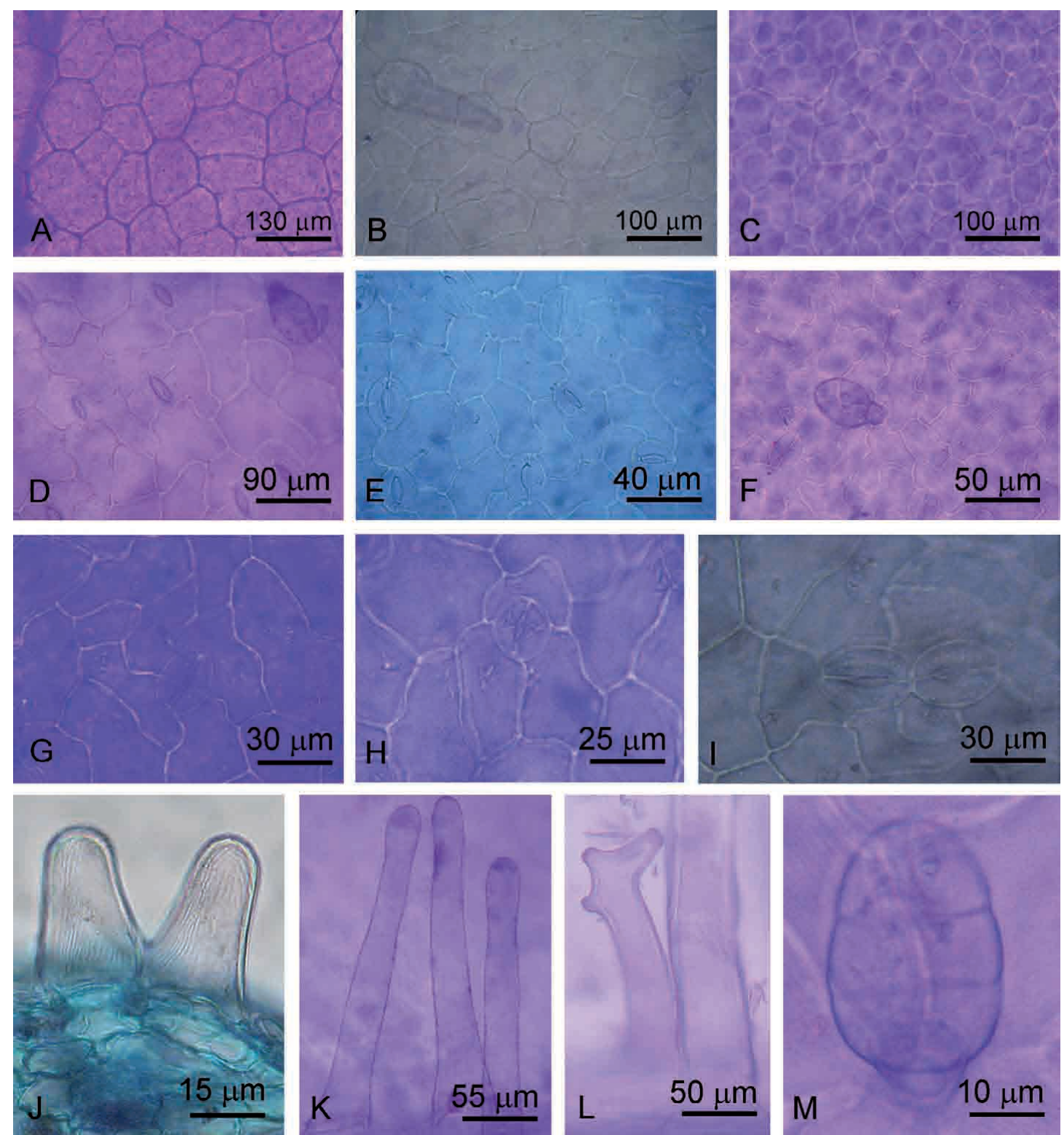

Fig. 2. Anatomía foliar. A, D) Dioscorea glomerulata. B, E) Dioscorea haumanii. C, F) Dioscorea sp. A-C) Epidermis adaxial. D-F) Epidermis abaxial. G) Estoma anomocítico. H) Estoma polocítico. I) Estomas gemelos. J) Tricoma eglandular unicelular corto. K) Tricoma eglandular unicelular largo. L) Tricoma eglandular unicelular largo ramificado. M) Tricoma glandular.

pluricelular compuesta por 6-8 células (Fig. 2M, Tabla 2). Ambos tipos de tricomas se distribuyen en las dos superficies.

En sección transversal, las tres entidades presentan lámina dorsiventral, hipostomática, y ambas epidermis unistratas, con cutícula gruesa ornamentada (Fig. 3A). El parénquima en empalizada presenta 2 estratos de células, el parénquima esponjoso está formado por 2-3 estratos (Fig. 3A, Tabla 2). Se observan cristales de oxalato de calcio en forma de drusas, prismas y rafidios (Fig. 3C, E, Tabla 2). Nectarios extraflorales se evidencian en la superficie adaxial de la lámina en D. glomerulata y Dioscorea sp. (Fig. 3D). En todas, las venas primarias y secundarias presentan haces vasculares colaterales, rodeados por una vaina parenquimática con casquete de 
Tabla 2. Caracteres diferenciales de la anatomía de la lámina de D. glomerulata, D. haumanii y Dioscorea sp.

\begin{tabular}{|c|c|c|c|c|}
\hline \multirow{2}{*}{\multicolumn{2}{|c|}{ Caracteres }} & \multicolumn{3}{|c|}{ Entidades } \\
\hline & & \multirow{2}{*}{$\begin{array}{l}\text { D. glomerulata } \\
\text { Isodiamétricas, } \\
\text { rectangulares, } \\
\text { cuadrangulares }\end{array}$} & D. haumanii & Dioscorea sp. \\
\hline $\begin{array}{l}\text { Forma de } \\
\text { las células }\end{array}$ & $\begin{array}{l}\text { Sup. } \\
\text { adaxial }\end{array}$ & & Isodian & רétricas \\
\hline epidérmicas & $\begin{array}{l}\text { Sup. } \\
\text { abaxial }\end{array}$ & \multicolumn{2}{|c|}{ Isodiamétricas } & Rectangulares \\
\hline \multicolumn{2}{|c|}{$\begin{array}{l}\text { Tamaño promedio de } \\
\text { estomas }(\mu \mathrm{m})\end{array}$} & $1,36+/-0,08$ & $1,38+/-0,10$ & $1,29+/-0,07$ \\
\hline \multicolumn{2}{|c|}{$\begin{array}{l}\text { Densidad estomática } \\
\left(\text { estoma } / \mathrm{mm}^{2}\right)\end{array}$} & $141,95+/-28,39$ & $125,31+/-47,02$ & $145,32+/-82,99$ \\
\hline \multicolumn{2}{|c|}{ Ubicación de estomas } & \multicolumn{2}{|c|}{$\begin{array}{c}\text { A nivel de las células epidérmicas o } \\
\text { elevados }\end{array}$} & $\begin{array}{l}\text { A nivel de las } \\
\text { células } \\
\text { epidérmicas }\end{array}$ \\
\hline \multicolumn{2}{|c|}{$\begin{array}{l}\text { Tipo y tamaño de } \\
\text { tricomas } \\
\text { eglandulares }(\mu \mathrm{m})\end{array}$} & $\begin{array}{c}\text { Largos } \\
\text { ramificados }(37,5 \\
+/-10,99) \\
\text { Largos sin } \\
\text { ramificar }(33,95 \\
+/-11,11)\end{array}$ & $\begin{array}{c}\text { Cortos }(19,5+/- \\
1,99) \text { y } \\
\text { largos }(29,23+/- \\
11,33)\end{array}$ & $\begin{array}{c}\text { Cortos }(21,5+/- \\
0,08) \text { y } \\
\text { largos }(31,95+/- \\
09,11)\end{array}$ \\
\hline \multicolumn{2}{|c|}{$\begin{array}{l}\text { Tamaño de tricomas } \\
\text { glandulares }(\mu \mathrm{m})\end{array}$} & $1,66+/-0,09$ & $1,75+/-0,09$ & $1,70+/-0,19$ \\
\hline \multicolumn{2}{|c|}{$\begin{array}{l}\text { Número de estratos de } \\
\text { parénquima esponjoso }\end{array}$} & \multicolumn{2}{|c|}{$3(4)$} & $2(3)$ \\
\hline \multicolumn{2}{|c|}{$\begin{array}{l}\text { Presencia de nectarios } \\
\text { extraflorales }\end{array}$} & Presentes & Ausentes & Presentes \\
\hline \multicolumn{2}{|c|}{$\begin{array}{l}\text { Tipos de cristales de } \\
\text { oxalato de calcio }\end{array}$} & Rafidios & $\begin{array}{c}\text { Rafidios, } \\
\text { drusas, prismas }\end{array}$ & Rafidios \\
\hline
\end{tabular}

esclerénquima hacia xilema y floema (Fig. 3B). Las venas primarias pueden poseer además una vaina esclerenquimática. También se observa presencia de colénquima o esclerénquima subepidérmico hacia ambas superficies. 


\section{Anatomía del pecíolo}

La anatomía del pecíolo es similar en las tres entidades estudiadas. En vista superficial ambas epidermis presentan células rectangulares a cuadrangulares de paredes rectas a curvas y cutícula estriada (Fig. $3 F$ ). Se observan estomas anomocíticos, raro braquiparacíticos (Fig. 3G), elevados o al mismo nivel de las células epidérmicas. Los tipos de tricomas son eglandulares y glandulares semejantes a los observados en la lámina (Fig. 2J-M), en D. glomerulata se observa un nuevo tipo de tricoma eglandular, pluricelular, ramificado (Fig. $3 \mathrm{H}$ ). En corte transversal el pecíolo tiene forma subcircular con borde irregular y una concavidad hacia la superficie adaxial (Fig. 3I). La epidermis es unistrata con cutícula estriada; hacia el interior le continúa colénquima laminar con 2 (3) estratos, seguido por parénquima con 3 (4) estratos donde se encuentran inmersos cinco haces vasculares con el xilema en forma de ' $\mathrm{Y}$ '. En algunas ocasiones se presentan seis o siete haces de menor tamaño hacia la superficie adaxial. Los haces vasculares poseen una vaina parenquimática y un casquete de esclerénquima interno (Fig. 3J).

\section{Análisis de Componentes Principales}

Los resultados del análisis de componentes principales en relación a la varianza explicada por cada uno de los componentes se muestran en la Tabla 3. Los dos primeros componentes, sintetizan el 65,5 \% de la variación total de los datos.

Del análisis de la matriz de correlación entre las variables originales y el primer componente (CP1) se deduce que las variables TH, DFM, ExF y TE se correlacionan con el mismo, TH, ExF y TE de manera positiva y la variable DFM de manera negativa. En el segundo componente (CP2) las variables TTG y DE tienen una correlación positiva y negativa respectivamente con el mismo (Tabla 4).

Lo antes expuesto se ilustra en la figura 4 mediante un gráfico de ordenamiento simultáneo de variables y entidades en el plano, formado por los componentes 1 y 2 . Se puede advertir que el CP1 separa a los ejemplares de las entidades estudiadas, ubicando hacia la derecha los que poseen mayor número de estambres por flor, tamaño de hojas y de estomas, mientras que hacia la izquierda ubica a los de valores menores para esos caracteres, es decir separa a D. glomerulata hacia la derecha, Dioscorea sp. hacia la izquierda y $D$. haumanii en valores intermedios. Por su parte el CP2 separa hacia el extremo superior a los ejemplares con mayor tamaño de tricoma glandular y mayor densidad estomática, y hacia el extremo inferior los de menor valor. En relación a esto, $D$. haumanii y $D$. glomerulata se ubican en la parte superior, mientras que Dioscorea sp. presenta una amplia variación entre los dos extremos.

\section{DISCUSIÓN Y CONCLUSIONES}

El presente trabajo analiza la relación entre D. glomerulata, D. haumanii y Dioscorea sp. en base a la morfología floral y la morfoanatomía foliar. 

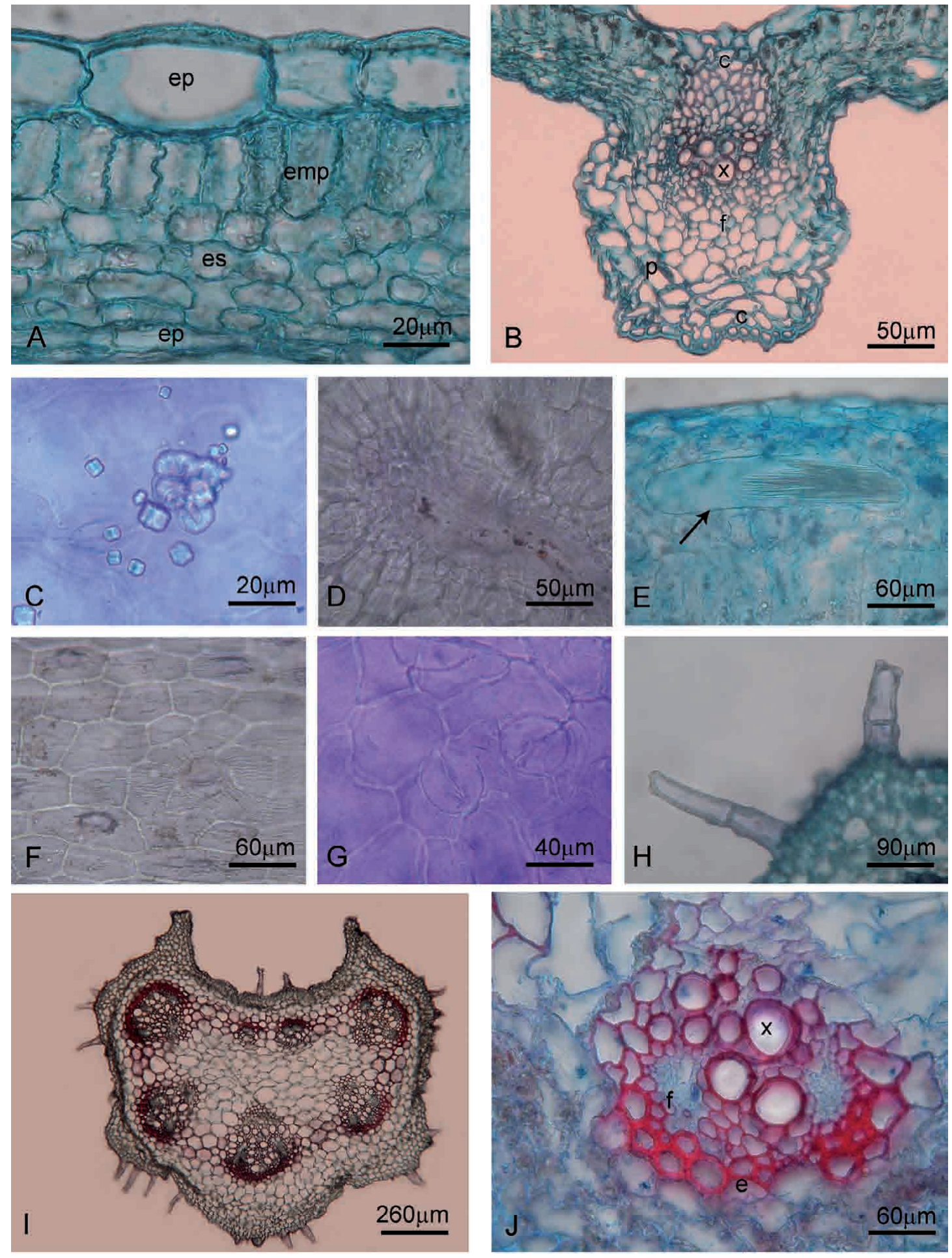

Fig. 3. Anatomía foliar. A) Sección transversal de la lámina. B) Sección transversal a la altura de la vena primaria. C) Cristales de oxalato de calcio en forma de prismas y drusas. D) Nectario extrafloral. E) Cristales de oxalato de calcio en forma de rafidios en idioblasto. F) Epidermis abaxial de pecíolo. G) Estoma braquiparacítico. H) Tricoma eglandular pluricelular ramificado. I) Sección transversal de pecíolo. J) Detalle de haz vascular. Referencias: ep, epidermis; emp, empalizada; es, esponjoso; c, colénquima; $x$, xilema; f, floema; e, esclerénquima; $p$, parénquima. 
Tabla 3. Autovalores y porcentaje de la variación explicada de los distintos componentes, calculados a partir de la matriz de Correlación R.

\begin{tabular}{cccc}
\hline Componentes & Valor Propio & \multicolumn{2}{c}{ Variación Total Explicada } \\
\cline { 3 - 4 } Principales & & Absoluta \% & Acumulada \% \\
CP1 & 2,47 & 41,22 & 41,22 \\
CP2 & 1,46 & 24,34 & 65,56 \\
CP3 & 0,99 & 16,51 & 82,07 \\
CP4 & 0,62 & 10,46 & 92,53 \\
\hline
\end{tabular}

El análisis de la morfología floral mostró caracteres diferenciales entre las tres entidades analizadas como el tamaño de la flor, la disposición y número de estambres. No se observaron caracteres intermedios entre Dioscorea sp. y las otras especies estudiadas.

Los rasgos morfológicos y anatómicos de las hojas de Dioscorea sp. y las especies estudiadas son consistentes con lo ya reportado para el género y la familia. Las entidades aquí estudiadas no evidencian elementos morfoanatómicos de valor diagnóstico que permita su delimitación como tal. Asimismo Dioscorea sp. no muestra caracteres intermedios entre $D$. glomerulata y $D$ haumanii. Sin embargo, hay algunos observados en este trabajo que merecen ser destacados ya sea por novedosos o porque reafirman reportes anteriores: la ausencia de venación intersecundaria observada sólo en Dioscorea sp., deberá ser cotejada con otras especies a fin de validar o no su exclusividad; la presencia de tricomas eglandulares en D. glomerulata, citada por primera vez para la Sección Dematostemon Griseb.; y los nectarios extraflorales presentes en Dioscorea sp. y D. glomerulata, carácter que podría interpretarse como indicador de proximidad entre ellas (Orr, 1926; Grout y Williams, 1980; Xifreda, 2000). La anatomía foliar de las especies de Dioscorea aquí estudiadas así como su morfología, revela caracteres semejantes a Monocotiledóneas y a Dicotiledóneas, como ser tipos de estomas, presencia de peciolo y tipo de nerviación. A este respecto, Huber (1998), al analizar caracteres anatómicos del tallo, postula que el género presenta un alto grado de aislamiento no sólo dentro de las Monocotiledóneas sino también en el resto de las plantas con semillas.

Tabla 4. Autovectores y correlación entre las variables y los componentes seleccionados. Referencias: DFM: diámetro flor masculina; DE: densidad estomática; TTG: tamaño tricoma glandular; TE: tamaño de estoma; TH: tamaño de hoja; ExF: estambres por flor.

\begin{tabular}{lcccc}
\hline \multirow{2}{*}{ Variables } & \multicolumn{2}{c}{ Componente Principal 1 } & \multicolumn{2}{c}{ Componente Principal 2 } \\
\cline { 2 - 5 } & Autovectores & $\begin{array}{c}\text { Coeficiente de } \\
\text { correlación } r\end{array}$ & $\begin{array}{c}\text { Autovectores } \\
\text { TH }\end{array}$ & $\begin{array}{c}\text { Coeficiente de } \\
\text { correlación } r\end{array}$ \\
TTG & 0,49 & 0,77 & 0,22 & 0,27 \\
DFM & $-0,16$ & $-0,25$ & 0,65 & 0,78 \\
ExF & $-0,54$ & $-0,85$ & 0,12 & 0,15 \\
DE & 0,56 & 0,88 & 0,01 & 0,01 \\
TE & 0,11 & 0,18 & $-0,62$ & $-0,75$ \\
\hline
\end{tabular}




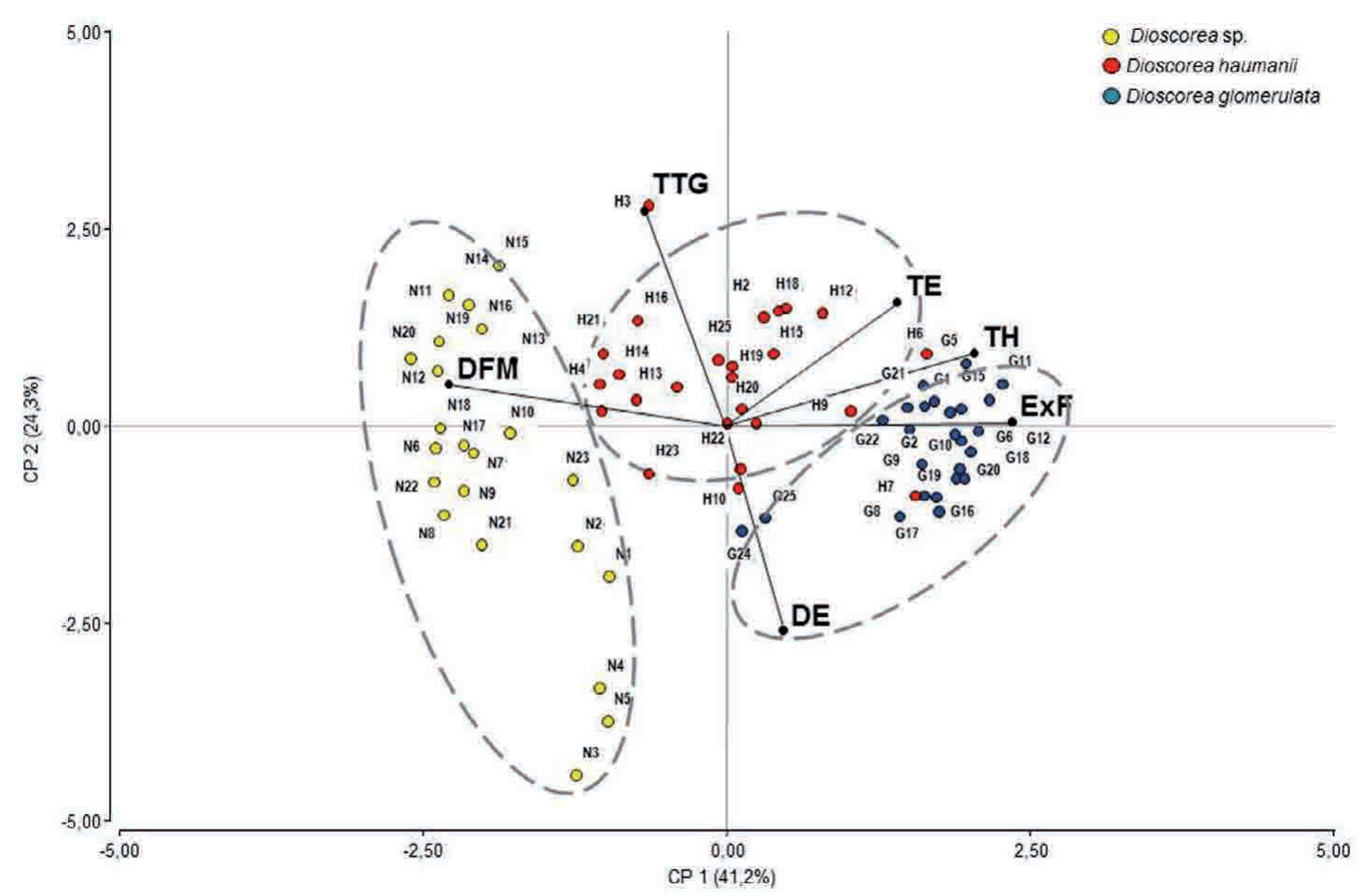

Fig. 4. Ordenamiento de los taxones de acuerdo a un Análisis de Componentes Principales de caracteres foliares y florales. Referencias: DFM: diámetro flor masculina; DE: densidad estomática; TTG: tamaño tricoma glandular; TE: tamaño de estoma; TH: tamaño de hoja; ExF: estambres por flor.

El ordenamiento del análisis estadístico muestra a Dioscorea sp. como una entidad independiente, alejada de los probables progenitores. Al analizar la distribución de puntos en Dioscorea sp., la misma refleja una variación intraespecifica en algunos de sus rasgos vegetativos, probablemente producto de la amplia distribución altitudinal y sus variadas condiciones ambientales asociadas (Burns, Cellini, Lencinas, Martínez Pastur, Rivera, 2010).

En lo que respecta a la presencia de un único estambre en Dioscorea sp. a pesar de ser un carácter controversial en el género, no necesariamente sería un indicio de hibridación. En efecto, D. monandra, una entidad con igual característica, fue recientemente reportada (Ulloa et al., 2017) como una especie válida. En relación a esto, Hauman (1916) consideró que la particularidad del androceo de esta especie, constituido por una columna única portando una única antera, podría interpretarse como una columna estaminal que representa los tres estambres primitivos reunidos en uno solo. Por otra parte, si bien las Monocotiledóneas fueron definidas, en parte, por la presencia de los ciclos florales en múltiplos de tres, actualmente se considera que esta característica es una condición ancestral y no una apomorfía (Soltis, Soltis, Endress, Chase, 2005).

En consecuencia y ante lo expresado, podemos concluir que este estudio posiciona a Dioscorea sp. en un lugar separado de D. glomerulata y D. haumanii lo que sugiere un origen independiente. Posteriores estudios genéticos, moleculares y de viabilidad serán necesarios para confirmar el origen y estatus taxonómico de esta entidad procedente de las Sierras de Calilegua (Jujuy). 


\section{AGRADECIMIENTOS}

Agradecemos a la Fundación Miguel Lillo por el espacio físico y equipamiento necesario que permitió concretar esta investigación, la que se enmarca en los proyectos B-0022-1 y B-0002-1 (Miguel Lillo 251, T4000JFE, Tucumán). También agradecemos a la Lic. Lelia Bordón de la sección Iconografía (FML), por la realización de las láminas de este trabajo y al Lic. Hugo Ayarde por sus aportes y correcciones del manuscrito.

\section{BIBLIOGRAFÍA / BIBLIOGRAPHY}

Abdulrahaman, A. A., Egbedo, F. O. y Oladele, F. A. (2009). Stomatal complex types, stomatal density, and the stomatal index in some species of Dioscorea. Archives of Biological Sciences 61 (4): 847-851. doi: 10.2298/ABS0904847A

Asesor, P. y Bulacio, E. (2017). Dioscorea naviculata (Dioscoreaceae), una nueva especie de origen híbrido de las Sierras de Calilegua (Jujuy, Argentina). XXXVI Jornadas Argentinas de Botánica. Mendoza. Argentina. Boletín de la Sociedad Argentina de Botánica 52 (supl.): 3.

Ayarde, H. (2013). Aporte hídrico de las neblinas en las montañas húmedas del noroeste de Argentina. I Simposio de la Reserva de Biósfera de las Yungas»10 años de experiencias y desafíos» y V Reunión de las Selvas de Montañas. Actas del I Simposio de la Reserva de Biosfera de las Yungas «10 años de experiencias y desafios» y V Reunión Regional de las Selvas de Montañas. 1:13. Jujuy: ISBN 978-950-721-448-6.

Ayensu, E. S. (1972). Dioscoreales. En: C. R. Metcalfe (Ed.), Anatomy of the Monocotyledon Vol. VI. (pp. 1-182). Oxford: Clarendon Press.

Barroso, G. M., Sucre, D., Guimarães, E. F., Carvalho, L. F., Valente, M. C., Silva, J. D., Da Silva, J. B., Rosenthal, F. R. T., Barbosa, C. M., Roseira, A. N., Barth, O. M. y Barbosa, A. F. (1974). Flora da Guanabara. Familia Dioscoreaceae. Sellowia 25: 9-256.

Barton, N. H. y Hewitt, G. M. (1985). Analysis of Hybrid Zones. Annual Review of Ecology and Systematics 16 (1): 113-148. doi: 10.1146 / annurev. es.16.110185.000553

Bulacio, E. y Ayarde, H. R. (2009). Flora y Vegetación del sector alto de las Sierras de Calilegua (Jujuy). XXXII Jornadas Argentinas de Botánica, Córdoba. Boletín de la Sociedad Argentina de Botánica. 44 (Supl.): 106.

Burkill, I. H. (1960). The organography and the evolution of Dioscoreaceae, the family of the yams. Botanical Fournal of the Linnean Society 56: 319-412.

Burns, S. L., Cellini, J. M., Lencinas, M. V., Martínez Pastur, G. J. y Rivera, S. M. (2010). Descripción de posibles híbridos naturales entre Nothofagus pumilio y N. antárctica en Patagonia Sur (Argentina). Bosque 31 (1): 9-16. doi: 10.4067/ S0717-92002010000100002 
Cabrera, A. L. (1976). «Regiónes fitogeográficas argentinas». En W. F. Kugler (Ed.), Enciclopedia Argentina de Agricultura y Fardinería. Tomo II, 2a . Edición (pp 1-85). Buenos Aires: Editorial ACME.

D’Ambrogio de Argüeso, A. (1986). Manual de Técnicas en Histología Vegetal. Buenos Aires, Argentina: Hemisferio Sur.

Daniel, A. y Atumeyi, S. (2011). Foliar epidermal anatomy of four species of Dioscorea. Avances in Applied Science Research 2 (4): 21-24.

Dilcher, D. L. (1974). Approaches to the identification of Angiosperm leaves. The Botanical Review 40 (1): 1-157. doi: 10.1007/BF02860067

Di Rienzo, J. A., Casanoves, F., Balzarini, M. G., Gonzalez, L., Tablada, M. y Robledo, C. W. (2012). Grupo InfoStat. FCA, Universidad Nacional de Córdoba, Argentina. Recuperado de http://www.infostat.com.ar

Dizeo de Strittmater, C. (1973). Nueva Técnica de diafanización. Boletín de la Sociedad Argentina de Botánica 15 (1): 126-129.

Ellis, B., Daly, D., Hickey, L., Johnson, K., Mitchell, J., Wilf, P. y Wing, S. (2009). Manual of leaf architecture. New York, USA: The New York Botanical Garden Press.

Govaerts, H. A., Wilkin, P. y Saunders, R. M. K. (2007). World Checklist of Dioscoreales: Yams and their Allies. London, England: Kew Publishing Royal Botanic Garden.

Grout, B. W. y Williams, A. (1980). Extrafloral nectaries of Dioscorea rotundata: their structure and secretions. Annals of Botany 46: 255-258.

Hauman, L. (1916). Les Dioscoréacées de L'Argentine. Anales del Museo Nacional de Historia Natural de Buenos Aires 27: 441-513.

Hickey, L. (1974). Clasificación de la arquitectura de las hojas de Dicotiledoneas. Boletín de la Sociedad Argentina de Botánica 16 (1-2): 1-26.

Hickey, L. (1979). A revised classification of the architecture of dicotyledonous leaves. En: C. Metcalfe, L. Chalk (Eds.), Anatomy of the Dicotyledons Vol. I. (pp. 25-39). Oxford: Clarendon Press.

Huber, H. (1998). Dioscoreaceae. In: K. Kubitzki (Ed.). The Families and Genera of Vascular Plants Vol 3, Flowering Plants-Monocotyledons. Lilianae (except Orchidaceae) (pp. 216-235). Berlin: Springer-Verlag.

Marín, E., Vargas, T. E. y Oropeza, M. (2012). Variabilidad genética y anatomía foliar comparada de plantas de Dioscorea alata mantenidas en cultivo in vitro. Interciencia 37 (6): 477-483.

Orr, M. Y. (1926). On the secretory organs of Dioscoreaceae. Notes from the Royal Botanic Gardens 15: 133-146.

Rushton, B. (1993). Natural hybridization within the genus Quercus L. Annales des sciences forestières 50 (1): 73-90.

Soltis, D. E., Soltis, P. S., Endress, P. K. y Chase, M. W. (2005). Phylogeny and Evolution of Angiosperms. Massachusetts, USA: Sinauer.

Ulloa Ulloa, C., Acevedo-Rodríguez, P., Beck, S., Belgrano, M. J., Bernal, R., Berry, P., Brako, L., Celis, M., Davidse, G., Gradstein, S., Hokche, O., León, B., León-Yánez, S. , Magill, R. E., Neill, D. A., Nee, M., Raven P. H., Stimmel, H., Strong, M. T., Villaseñor Ríos, J. L., Zarucchi, J. L., Zuloaga, F. O. y Jørgensen, P. M. 
(2017). An integrated assessment of vascular plants species of the Americas. Science 358: 1614-1617.

Xifreda, C. C. (1990). Sobre la presencia de una vaina cristalìfera en el tallo de algunas especies de Dioscorea (Dioscoreaceae). Darwiniana 30 (1-4): 137-142

Xifreda, C. C. (1996). Dioscoreaceae. En: F. O. Zuloaga, O. Morrone (Eds.), Catálogo de las Plantas Vasculares de la República Argentina I. Monographs in Systematic Botany from the Missouri Botanical Garden 60: 197-202.

Xifreda, C. C. (2000). Evaluation of pollen and vegetative characters in the systematics of South American species of Dioscorea (Dioscoreaceae) In: K. L. Wilson, D. A. Morrison (Eds.), Systematics and evolution of monocots (pp. 488-496). Melbourne: CSIRO.

Xifreda, C. C. y Seo, M. (2009) Dioscoreaceae. En: L. J. Novara (Ed.), Aportes Botánicos de Salta, Flora del Valle de Lerma. (22 pp). Salta: Herbario MCNS, Facultad de Ciencias Naturales, Universidad Nacional de Salta.

Zobel, B. y Talbert, J. (1994). Técnicas de mejoramiento genético de árboles forestales. Noriega, México: UTEHA. 